\title{
Minimal mechanistic component of proteasome activation and prevention of impairment by pathological oligomers
}

Janelle Chuah

West Virginia University https://orcid.org/0000-0002-1139-694X

Tifffany Thibaudeau

West Virginia University

David Smith ( $\square$ dmsmith@hsc.wvu.edu )

West Virginia University https://orcid.org/0000-0002-1502-676X

\section{Article}

Keywords: proteasome, biochemistry, $\mathrm{HbYX}$

Posted Date: September 23rd, 2021

DOI: https://doi.org/10.21203/rs.3.rs-900719/v1

License: (1) (1) This work is licensed under a Creative Commons Attribution 4.0 International License.

Read Full License 


\section{Abstract}

Impairment of proteasomal function has been implicated in neurodegenerative diseases, justifying the need to understand how the proteasome is activated for protein degradation. Here, using biochemical and structural (cryo-EM) strategies in both archaeal and mammalian proteasomes, we further determine the $\mathrm{HbYX}$ (-motif)-dependent mechanism of proteasomal activation used by multiple proteasome-activating complexes including the 19S Particle. We identify multiple proteasome a subunit residues involved in $\mathrm{HbYX}$-dependent activation, a point mutation that activates the proteasome by partially mimicking a $\mathrm{HbYX}$-bound state, and conformational changes involved in gate-opening with a 2.0A structure. Through an iterative process of peptide synthesis, we successfully design a $\mathrm{HbYX}$-like dipeptide mimetic as a robust tool to elucidate how the motif autonomously activates the proteasome. The mimetic induces near complete gate-opening at saturating concentration, activating mammalian proteasomal degradation of peptides and proteins. Findings using our peptide mimetic suggest the HbYX-dependent mechanism requires cooperative binding in at least two intersubunit pockets of the a ring. Collectively, the results presented here unambiguously demonstrate the lone role of the HbYX tyrosine in the allosteric mechanism of proteasome activation and offer proof of concept for the robust potential of HbYX-like small molecules to activate the proteasome.

\section{Introduction}

The proteasome is a key component of the ubiquitin-proteasome system (UPS), responsible for removing damaged or unneeded proteins and regulating major cellular processes ${ }^{1}$. Regulation of the proteasome is key to this function to ensure that only damaged \& unnecessary proteins are degraded. Dysregulation of the proteasome has been implicated in several neurodegenerative diseases (NDs), often characterized by impairment of proteasome function ${ }^{2-7}$. In this study, we elucidate a mechanism of proteasomal activation and design a small molecule to functionally emulate the mechanism of proteasomal activation. Deriving from our findings on the mechanism of proteasomal activation, we report that our small molecule robustly regulates the activity of the archaeal, yeast, mammalian, and human proteasomes, and are even able to overcome the inhibition of the proteasome by protein aggregates implicated in neurodegenerative disease, such as amyloid- $\beta$ and $\alpha$-synuclein.

The Core Particle of the proteasome, also referred to as 20S (Fig. 1A), consists of four stacked heteroheptameric rings ( $\alpha-\beta-\beta-\alpha)$ with a central pore for substrate entry. The $\beta$ rings consist of seven subunits ( $\beta 1-7)$, three of which harbor the protease sites. The two a rings also consist of seven subunits (a1-7). Substrate entry in the 20S is regulated by the gate, which primarily consists of the N-terminus of a 2,3 , and 4 extending over the central pore ${ }^{8}$. The closed gate conformation blocks the central pore and prevents proteins or peptides from entering the $20 \mathrm{~S}$ to be degraded. The $\mathrm{N}$-terminus of each a subunit carries a YDR motif that interacts with neighboring $\mathrm{N}$-termini to stabilize the closed state of the gate $\mathrm{e}^{8}$. These N-termini extensions can also change their conformation to an "open" state, whereby they point up and outwards from the a ring pore, which is stabilized by an alternative interaction from the YDR motif ${ }^{9}$. 
Assessed by NMR, the basal kinetics of archaeal 20S gate-opening has been suggested to undergo open/close fluctuations on a time scale of seconds ${ }^{10,11}$. Although the kinetics have not yet been measured for the mammalian 20S, the presence of basal protein or peptide hydrolysis activities suggest that the mammalian 20S gate also fluctuates between these states, though likely slower. Additionally, truncation of a3 $\mathrm{N}$-terminus ( $\mathrm{a} 3 \Delta \mathrm{N}$ ), which act as a central lynchpin to stabilize the closed state, generates a constitutively open (active) $20 \mathrm{~S}$ that is highly capable of degrading unstructured proteins $\mathbf{s}^{8,12}$.

While the 20S gate is typically in the closed state, binding of proteasome regulatory complexes (Fig. 1A) to the a ring can trigger conformational changes that cause gate-opening, allowing the $20 \mathrm{~S}$ to accept linearized substrates ${ }^{13,14}$. Two different mechanisms of $20 \mathrm{~S}$ gate-opening have been described, the $\mathrm{HbYX}$-dependent and the 11S family-dependent mechanisms. Thus far, the majority of studies on the HbYX-dependent mechanism focuses on the 19S, also known as PA700 or the Regulatory Particle (RP), which associates with the $20 \mathrm{~S}$ to form the $26 \mathrm{~S}$ complex that degrades ubiquitinated proteins (Fig. 1A). The 19S consists of a base subcomplex, which is primarily composed of a heterohexameric ring of ATPases (Rpt1-6), and a lid subcomplex, which contains ubiquitin binding and processing subunits. The 19S has been shown to interact with the 20S and stimulate gate-opening by the docking of the $\mathrm{C}$-terminal tails of Rpt1-6, some of which contain the HbYX (hydrophobic-tyrosine-almost any C-terminal residue) motif, in the intersubunit pockets of the 20S a ring ${ }^{15}$. The use of C-terminal tails to associate with the $20 \mathrm{~S}$ has also been observed in other Proteasome Activators (PAs) including PAN (proteasome-activating nucleotidase; the archaeal homolog of the 19S), PA200/BIm10 (Fig. 1A), and 11S activators.

Not all C-terminal tails of PAs induce gate-opening when bound to the 20S, as evident by the observation that peptides corresponding to the C-terminus of PA26 (a member of the 11S family) cannot induce gateopening autonomously ${ }^{16}$. Conversely, peptides corresponding to the C-terminus of Rpt2, Rpt3, Rpt5, PAN, and PA200/BIm10 can induce autonomous gate-opening ${ }^{16,17}$. All these peptides that induce gate-opening carry the HbYX motif, which has been shown to be essential for allowing these complexes to associate with the 20S ${ }^{15,16}$. The C-terminal HbYX motif binds into pockets formed by the interface of the a subunits in the 20S, called intersubunit pockets (Fig. 1B\&C). Whereas the C-termini of PAN homohexameric ATPases all have a HbYX motif, only the C-terminus of Rpt2, 3, \& 5 from the $19 \mathrm{~S}$ heteromeric ATPases have the $\mathrm{HbYX}$ motif and Rpt1 has a partial $\mathrm{HbYX}$ motif, lacking the $\mathrm{Hb}$ residue. The roles that the $\mathrm{C}$ termini of Rpt4 and Rpt6 (which lack the HbYX motif) play in the association of the 19S-20S and 20S gating regulation are unclear but have been seen bound to intersubunit pockets via cryo-EM ${ }^{18,19}$. The binding of $\mathrm{HbYX}$ peptides to intersubunit pockets, structurally distant from the gating residues, results in gate conformational change. This demonstrates that the $\mathrm{HbYX}$ motif functions allosterically, and likely induce substantial conformational changes in the a subunits that in turn, affect the conformation of gating residues ${ }^{16,20}$.

In contrast, the family of 11S activators does not have a HbYX motif on their C-terminal tails. Their mechanism of gate-opening is relatively well-known compared to the HbYX-dependent mechanism. They associate with the 20S using their C-termini to dock in the a intersubunit pockets, similar to the HbYX- 
dependent activators. However, to trigger gate-opening, the 11S family rely on "activation loops" that interface directly with the base of the gating $\mathrm{N}$-termini in the pore of the a ring ${ }^{21,22}$. These activation loops appear to sterically repel a reverse turn proline (Pro17) at the base of the gating residues, which destabilize the closed state and stabilize the open state. Interestingly, relative to the HbYX-mechanism, minimal conformational changes in the a subunits (excluding gating regions) are necessary for gateopening by the $11 \mathrm{~S}$ activators, as shown by the crystal structure of PA26-20S proteasome ${ }^{9}$. It is evident that the two families of PAs (HbYX-dependent and HbYX-independent) use different strategies to induce $20 \mathrm{~S}$ gate-opening. Although the location and effect of $\mathrm{HbYX}$-binding has been investigated, the molecular mechanism of $\mathrm{HbYX}$-dependent gate opening appears to be surprisingly complex and remains unsolved.

Structures of the substrate-engaged human 26S (H26S) from Dong et. al ${ }^{18}$ suggest that the human 19S (H19S) associates with the human 20S (H20S) through various interactions between the 19S ATPase's Ctermini and the $20 \mathrm{~S}$ a-ring. These interactions vary in that they change based on the state of the $26 \mathrm{~S}$. As the $\mathrm{H} 26 \mathrm{~S}$ transition towards a more active state $\left(\mathrm{E}_{\mathrm{A} 1,2}>\mathrm{E}_{\mathrm{B}}>\mathrm{E}_{\mathrm{C} 1,2}>\mathrm{E}_{\mathrm{D} 1,2}\right)^{18}$, more C-termini form stable interactions (as observed via cryo-EM), starting with Rpt3, Rpt5 and Rpt2, then Rpt6, and finally Rpt1. The first tails to dock (Rpt3, 5, \& 2) all carry the HbYX motif, yet, as visualized by cryo-EM the gate does not appear open. When the last C-terminus of the ATPases binds (Rpt1, which has a partial HbYX motif), a conformational change occurs, resulting in a stably opened gate. Interestingly, another structural study of Saccharomyces cerevisiae 26S (Y26S) ${ }^{23}$ suggests the same pattern of C-terminal tail binding for gate opening, while other studies on the $\mathrm{Y} 26 \mathrm{~S}^{19,24}$ suggest complete gate-opening occurs after the binding of Rpt6, and that the binding of Rpt 1 in the a ring is not required for complete gate-opening. Additionally, some Y26S structures ${ }^{19,24}$ indicate that the binding of Rpt2, 3, \& 5 alone is sufficient to induce partial gate-opening. Li et. al. previously demonstrated via a biochemical study that the mammalian $20 \mathrm{~S}$ gate was "variably modulated" when complexed with the 19S, and could exist in various states of openness. While cryo-EM findings can approximate gate openness, the structures do not precisely reflect the dynamics of the gate within an individual proteasomal state $\left(\mathrm{E}_{\mathrm{A} 1,2}, \mathrm{E}_{\mathrm{B},} \mathrm{E}_{\mathrm{C} 1,2}, \mathrm{E}_{\mathrm{D} 1,2}\right)$. For example, the unactivated $20 \mathrm{~S}$ proteasome by itself still has the ability to degrade linearized proteins or peptides, but structurally, it is observed with a closed gate via cryo-EM or X-ray crystallography. Therefore, structural methods have a limited ability to draw conclusions regarding the functional openness of a gate.

The disparity between the HbYX-dependent mechanism of gate opening in the H26S and Y26S raises the question of how the HbYX-motif binding opens the gate. It is possible that $\mathrm{HbYX}$-dependent gate opening requires occupation of a minimum number of intersubunit pockets to trigger an allosteric transition. Alternatively, it's possible that only a subset of pocket(s) must be occupied by the corresponding C-termini to trigger gate-opening, or some combination of these two scenarios. We expect that since the HbYX motif is conserved from archaea to humans and is conserved in most PA's (excluding the 11S family), the $\mathrm{HbYX}$-dependent mechanism of gate-opening would also be conserved.

Recently, we showed that ND-associated proteins (i.e. amyloid-beta, a-synuclein, and huntingtin) can fold into a common conformation that inhibits the $20 \mathrm{~S}$ and $26 \mathrm{~S}$ proteasomes ${ }^{20}$. The inhibitory conformation 
is recognized by the $\mathrm{A} 11$ antibody, using a conformational epitope known to be pathological ${ }^{25}$. We also learned that these A11-positive (A11+) oligomers inhibit the $20 \mathrm{~S}$ by allosterically stabilizing the closed gate conformation ${ }^{20}$. This negative allosteric regulation by the $\mathrm{A} 11+$ oligomers appear to be mechanistically coupled to the $\mathrm{HbYX}$-dependent mechanism of $20 \mathrm{~S}$ gate-opening ${ }^{20}$, suggesting that $\mathrm{A} 11$ + oligomers and the $\mathrm{HbYX}$ motif are allosteric regulators of the same gating mechanism. This leads us to hypothesize that stimulating $20 \mathrm{~S}$ activity via the $\mathrm{HbYX}$-dependent mechanism could not only antagonize impairment by $\mathrm{A} 11$ + oligomers and restore proteasome function, but it could also stimulate protein degradation, potentially providing a therapeutic approach for ND. Whereas the molecular interactions that stabilize the closed (i.e. 20S-a subunit YDR motif) and open state of the proteasome's gate are clear ${ }^{8,26}$, as are the various $26 \mathrm{~S}$ proteasome opened/closed states ${ }^{18,19,23,24}$, the molecular mechanisms that regulate the transition between these closed and open states are not understood. A clear understanding of the gate-opening mechanism in the $20 \mathrm{~S}$ will provide the molecular framework to guide drug-discovery approaches aimed at activating proteasomal degradation to treat ND and is expected to elucidate how ND-associated oligomers impair proteasome function.

\section{Results}

Identifying essential structures for HbYX dependent gate-opening.

The HbYX motif is highly conserved and found in archaeal, yeast, and human proteasome activators, among other species. Through mutagenesis and structural studies, we found that the intersubunit pockets contain multiple conserved residues, some of which are already known to be important for gate activation (i.e., Pro17, Lys66) 9,16,27 (Fig. 2A). To better understand how the HbYX motif interacts with the 20S, we aligned HbYX-bound intersubunit a pockets from human (PDB 6msk, cryo-EM), yeast (PDB 4v7o, crystallography), and archaeal proteasomes (PDB 6hed, cryo-EM) (Fig. 2B). We observed in the mentioned structures that the $\mathrm{HbYX}$ motif binds to all three species of intersubunit pockets in similar orientations, with three distinct interactions (Fig. 2B): (1) The C-terminal carboxylic acid of the motif is directed towards the conserved lysine (K66 in archaea), which is already known to be required for $20 \mathrm{~S}$ proteasome-activator complex formation, (2) the penultimate tyrosine's hydroxyl group hydrogen-bonds with the backbone carbonyl of G19 and is oriented toward the proline reverse turn (located at the base of the gate), and (3) the hydrophobic $(\mathrm{Hb})$ group contacts a hydrophobic pocket in the neighboring a subunit.

We investigated the effect of mutating three of the conserved residues that are positioned to interact with the HbYX-motif (Fig. 2C) in the Thermoplasma acidophilum 20S (T20S). We chose this protein because it is a homoheptamer, which facilitates simultaneous mutagenesis in all 7 intersubunit pockets. We then tested activation of the T20S mutants by PAN (measuring LFP peptide degradation) since PAN uses the $\mathrm{HbYX}$-dependent mechanism (Fig. 2C). Consistent with previously published results ${ }^{16}, \mathrm{~K} 66 \mathrm{~A}$ mutation prevented PAN activation of the T20S. The basal activity of the T20S-K66A was similar to the wild type (WT). Structural studies have shown that the aliphatic portion of the K33 side chain and L81 are 
positioned on either side of $\mathrm{HbYX}$ tyrosine ring, interacting hydrophobically to hold the HbYX tyrosine ring in place (Fig. 2B). When K33 was mutated to glycine, the mutant T20S could no longer be stimulated by PAN (Fig. 2C). Also, the T20S-K33G basal activity was about half of the wild-type. Similarly, the mutation, L81A (located directly under the HbYX tyrosine) prevented PAN-mediated proteasome activation.

Therefore, based on prior structures and these biochemical results, we conclude that K33 and L81 are both important for stabilizing and, likely, orienting the HbYX tyrosine in the intersubunit pocket.

Considering the importance of $\mathrm{HbYX}$ tyrosine hydrogen-bonding with the backbone carbonyl of $\mathrm{G} 19$, we investigated whether substituting tyrosine for L81 (L81Y) could mimic the effect of a bound HbYX motif, as the mutation would place a tyrosine ring in similar space as the HbYX tyrosine (Fig. 2E). We found that proteasomes with the L81Y mutation (T20S-aL81Y) had elevated basal activity, relative to WT (Fig. 2D), suggesting that the mere placement of a tyrosine into this location is sufficient to induce gate opening, similar to $\mathrm{HbYX}$ binding. In agreement with this conclusion, addition of PAN at saturating concentration to T20S-aL81Y proteasomes only resulted in a $30 \%$ increased activation to near WT-like activation (Fig. 2D). These results demonstrate that the proper placement of a tyrosine alone in all intersubunit pockets of the archaeal $20 \mathrm{~S}$ proteasome is sufficient to induce conformational changes leading to significant 20S gate opening.

\section{Analyzing the conformational changes of L81Y in T20S}

Using cryo-EM, we determined the structure of the L81Y T20S. We also investigated the conformational changes induced by the L81Y mutation that resulted in the observed increased basal activity (Fig. 2D). From $\sim 890,000$ single particles we were able to generate a $2.1 \AA \AA$ cryo-EM reconstruction. The resolution was sufficient to confirm the L81Y mutation (Fig. 3A) in the a intersubunit pockets. Notably, the space occupied by the side chain of WT T20S L81 is replaced with a larger density in L81Y T20S, indicative of the greater size of tyrosine's side chain relative to leucine's. To determine the conformational changes in L81Y T20S that increased its peptide hydrolysis activity (Fig. 2D), we compared our L81Y map against the WT T20S. Previously, Campbell et $\mathrm{al}^{28}$ published the cryo-EM structure of the WT T20S at $2.8 \AA$ resolution. From the same published data set (EMD-6287), we reconstructed a $2.6 \AA$ structure using

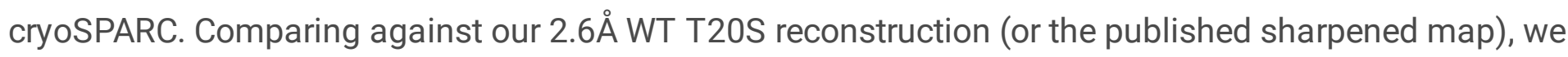
observed obvious conformational changes primarily in the a rings, with minor change observed

in the $\beta$ subunits. Specifically, we noted an expansion of what we refer to hereon as the "Pro17 Ring", (Fig. 3B, top view, SMovie 1). This Pro17 ring expansion is made up of all 7 symmetric Pro17's moving in a radial direction away from the central pore. In addition, these Pro17s extend upwards away from the $\beta$ subunits, slightly lengthening the 20S. (Fig. 3B, SMovie 2). The expansion of the Pro17 ring was also concomitate with larger movements of helices 0 and 3 , which also move primarily away from the central pore when viewed form the top (Fig. 3B, SMovie 1). It's understood that the YDR motif on the N-terminal of the alpha subunits is important to stabilize the closed and open states of the gate in the eukaryotic 20S, and has been shown to stabilize the open state in the T20S. However, the role of the YDR motif is not as well understood in the closed state of the T20S, due to its dynamic nature. Based on the Pro17 ring 
expansion seen in the L81Y mutant we hypothesize that this expansion "pulls" the N-termini outward disrupting the dynamic interactions in the pore, causing the gate to open.

Since observing that the L81Y T20S has an elevated basal activity (Fig. 2D), we also analyzed the conformation of the gating densities in our reconstruction. It's well understood that the gating residues in the archaeal 20S proteasome are dynamic and produce "fuzzy" densities in the pore of the T20S. The process of sharpening or density modification therefore results in some expected loss of density corresponding to the gate. Therefore, we also analyzed the gating densities using unsharpened maps (Fig. 3C) at normalized threshold levels that generated equally sized secondary structure densities in the $\beta$ subunits, which are the least dynamic. At these normalized threshold levels, we noticed a distinct difference in the shape and density of the gating residues in the pore. The pore of the WT was occluded by a tall and narrow dome-shaped density (Fig. 3C). The dome-shaped density occupying the WT T20S pore suggests that the $\mathrm{N}$-terminal tails of a subunits can extend upwards, leaning towards the center to interact with each other thus "closing" the gate. On the contrary, the pore of the L81Y map is open and is occupied with far less density. In addition, the observed density in L81Y's gate has a very different topology, suggesting the $\mathrm{N}$-termini are interacting in a manner that could form a ring around the channel similar to the aperture of a camera. In addition, at lower threshold levels that show more density in the map, the gating dome in the L81Y $20 \mathrm{~S}$ is much broader and shorter than the WT gating densities (SFig. 1, SMovie 3). We take these observations to be indicative of gate-opening in the L81Y-T20S. Using these normalized threshold levels, we generated a morph between the WT and L81Y T20S structures showing the a subunit conformational changes combine with the opening of the gate in the unsharpened map. While we previously presumed that in the open conformation, the N-terminal tails dynamically move above the a ring, our map suggests the $\mathrm{N}$-terminal tails may form a ring-like structure that constitutes the open state (Fig. 3C), though this remains speculative based on the inherent disorder of this region. Collectively, our cryo-EM reconstructions suggests that the single residue mutation, L81Y, induces conformational changes that expand the entry channel and favors the open gate conformation.

\section{Generating a minimal HbYX motif-based peptide to stimulate gate opening.}

Since the L81Y mutation was able to induce gate opening, we hypothesized that small peptides mimicking the HbYX-motif could activate 20S gate opening. Our previous study in 2007 showed that only peptides $>7$ residues long with a C-terminal HbYX motif (corresponding to the PAN's C-terminus) could induce gate opening ${ }^{16}$. However, when this length dependence was initially tested, peptides $<7$ residues in length were synthesized with unmodified N-termini. We suspected that the charged $\mathrm{N}$-terminus may prevent shorter $\mathrm{HbYX}$ peptides from binding to the intersubunit a pockets. To test this hypothesis, we synthesized peptides corresponding to the PAN C-terminus from three to eight residues in length (CT3-8), except with an acetylated $\mathrm{N}$-terminus. In contrast to previous results, $\mathrm{N}$-acetylated PAN C-terminus peptides shorter than seven residues stabilized the open 20S gate (Fig. 4A). Notably, we observed that Nacetylated PAN C-terminus peptide six residues in length (CT6) activated the $20 \mathrm{~S}$ over 10 -fold greater than the control; even the 3-residue peptide (CT3) had significant ( 2-fold) gate-opening activity. 
Based on the successful gate-activation of PAN CT3 (Ac-LYR) after a single modification, we investigated whether additional modifications would further improve the efficacy of the peptide. Our previous study showed that the substitution of alanine for arginine does not affect PAN-CT8 peptide efficacy to bind a intersubunit pockets and open the gate ${ }^{16}$. We therefore substituted an alanine in place of the bulky arginine residue, which decreased the size of the tripeptide and resulted in greater activating efficacy, compared to Ac-LYR (Fig. 4B). Attempting to further minimize the size of the molecule, we chose carboxybenzyl (Z) as an N-terminal blocking group that could eliminate the N-terminal charge and mimic the $\mathrm{Hb}$ group (of the HbYX motif). Combining these modifications resulted in a dipeptide with a hydrophobic group preceding the N-terminus of tyrosine (Z-Tyr-Ala or ZYA) (Fig. 4C). We compared ZYA against Ac-LYR and Ac-LYA and observed significant improvements in T20S activation (Fig. 4B). ZYA could substantially activate T20S activity, but its affinity was poor (see below). While ZYA could activate T20S 14 fold, it could not stimulate gate-opening in T20S-K66A to any extent (Fig. 4D), demonstrating that ZYA requires $\mathrm{K} 66$ in the intersubunit pocket, which is known to be required for the binding of PA's Ctermini to these pockets. This suggests that activation of T20S by ZYA occurs through interactions similar to those responsible for activation by PAN. To rule out the possibility that ZYA might be activating the protease sites rather than inducing gate opening, we also tested the capacity of ZYA to stimulate the $\mathrm{a} \Delta \mathrm{N}-\mathrm{T} 20 \mathrm{~S}$, which lacks gate-residues and is therefore constitutively open. ZYA did not stimulate the activity of $a \Delta \mathrm{N}$-T20S to any extent (Fig. 4E). Together, these results demonstrate that ZYA activates the $20 \mathrm{~S}$ by inducing gate-opening, similar to the HbYX motif.

\section{ZYA activates mammalian proteasomes in a cooperative fashion.}

Next, we tested Ac-LYR, Ac-LYA, and ZYA on mammalian 20S. As expected, we observed significant improvements in gate-opening efficacy with each modification (SFig. 1), similar to T20S. ZYA exhibited the greatest effect on the mammalian 20S, showing near 50-fold activation at 5mM (Fig. 5A). Although ZYA can stimulate the mammalian $20 \mathrm{~S}$ activity robustly, it has low affinity with a $\mathrm{k}_{\text {obs }}$ of $\sim 1 \mathrm{mM}$ (Fig. 5A). To confirm that ZYA specifically affects gate opening in eukaryotic proteasomes, we tested ZYA on yeast open-channel mutant (a3 $\Delta N$ ) 20S and observed that only WT Y20S, but not the a3 $\Delta N$ Y20S, could be activated by ZYA (Fig. 5B), consistent with our experimental results using the archaeal proteasomes. ZYAinduced proteasome activity in mammalian $20 S$ showed a cooperative binding curve with a hill coefficient of $1.5+/-0.1$ (Fig. 4A), indicating that ZYA binding is cooperative and thus likely binds to more than one site before inducing allosteric changes that result in gate-opening. This is consistent with published Cryo-EM structures of the $26 S^{18,19}$ that showed three or four C-termini binding before the opengate state is fully stabilized.

We next tested the ability of ZYA to stimulate the mammalian $20 S$ to hydrolyze three different peptide substrates that are preferentially cleaved by 20S's three different protease sites (LLVY-amc, $\beta 5$; nLPnLDamc, $\beta 1$; LRR-amc, $\beta 2$ ). We observed a significant and dramatic (10- to 50-fold) increase in the hydrolysis rate of all three peptide substrates (Fig. 5C, compared to DMSO), as expected for a compound that regulates gate opening. In addition, to determine how well ZYA was able to induce gate-opening, we compared it to 20 S activation by PA26. Saturating PA26 concentrations typically stimulate 20S activity 
by 30-100-fold, depending on the basal activity of the 20 S preparation. We found that ZYA was able to stimulate the 20S similar to that of PA26: 50-fold activation by ZYA and 90-fold for PA26. We also combined ZYA and PA26 to show that there was no synergy between these activators, as expected if they both function by inducing gate opening. When ZYA and PA26 are combined, there is a slight decrease in activity, relative to PA26 alone, which is likely due to competition between ZYA and PA26 for binding to the intersubunit pockets. Collectively, these results demonstrate that ZYA is a highly effective, though low affinity activator of gate opening in $20 \mathrm{~S}$ proteasomes from archaea and eukaryotes.

\section{ZYA stimulates protein degradation by the mammalian 20 s proteasome}

While we have demonstrated that ZYA robustly activates peptide hydrolysis via gate-opening, changes in peptide degradation activity do not necessarily reflect changes in the 20S's capacity to degrade unstructured proteins. To determine if ZYA has the capacity to stimulate protein degradation of tau23 (a truncated tau protein) and casein, we analyzed degradation by SDS-PAGE. ZYA significantly increased the degradation of both proteins, as visualized by Coomassie stain (Fig. 5D). Density differences between substrate and $20 \mathrm{~S}$ bands (loading control) show the substrate concentration to be saturating. Tau and casein appeared to be completely degraded within the first $15 \mathrm{mins}$ and $30 \mathrm{mins}$, respectively. In support of these results, we also measured the peptides generated from the degradation of ${ }^{14} \mathrm{C}$-casein by the $20 \mathrm{~S}$ in solution (Fig. 5E) as measured by acid-soluble counts. In agreement with the gel-based protein degradation assay, ZYA significantly increased the number of soluble peptides from the degradation of ${ }^{14}$ C-casein.

\section{Probing structure activity relationships of the ZYA gate- opening compound}

To elucidate how ZYA might be binding to the proteasome, we computationally docked ZYA in the human a5/6 intersubunit pocket, where the HbYX motif of Rpt5 binds. Our docking results (Fig. 6A) suggests that ZYA binds in a similar configuration to the HbYX motif of various PAs (Fig. 2B). We proceeded to probe ZYA's structure/function relationships and efficacy through chemical modifications ( Fig. 6B\&C). First, we asked if additional negative charges in the " $X$ " position of the HbYX motif could be tolerated by replacing alanine with acidic residues. We found that ZYE and ZYD failed to activate the 20S (Fig. 6B). We also tested if causing a backbone torsion constraint (ZYP) and a polar group (ZYQ) might stabilize the peptide's structure and binding, but they too abrogated activity and did not activate the mammalian $20 \mathrm{~S}$ proteasome (Fig. 6B). Prior studies and sequence conservation showed that small, medium, and large aliphatic, and basic residues could all be tolerated in the " $X$ " position, thus the " $X$-almost any residue" designation ${ }^{16}$. However, our data suggests there are some limitations (i.e., negative charges) in this position. We also attempted to block the C-terminal negative charge by modifying the charged carboxylic acid on ZYA with $\mathrm{NOH} 2$ group. We observed that ZYA-[NOH2] failed to stimulate the proteasome (Fig. 6B), suggesting that the charged $\mathrm{C}$-terminus is critical for $\mathrm{HbYX}$ to mediate gate activation, as would be expected if it forms a salt bridge with $\mathrm{K} 66^{16}$. 
Since hydrogen bonding between the HbYX hydroxyl group and the G19 carbonyl (near the proline reverse turn) is thought to be important for gate activation by $\mathrm{HbYX}$-containing PAs and $\mathrm{C}$-terminal peptides, we

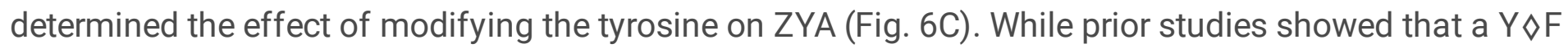
mutation in the HbYX motif of PAN or 8 residue C-terminal peptides abrogated gate-opening ${ }^{16}$, we find that the shorter tripeptide Ac-YFA activated the 20S proteasome gate, though to a lesser extent than ZYA (Fig. 6D). It is worth noting that some eukaryotic PAs have a YYA HbYX motif (i.e., human Rpt5, yeast Blm10), hence it is perhaps not surprising that activation could be obtained by Ac-YFA. The ability of AcYFA to activate the proteasome gate suggests the intersubunit binding pocket can accommodate a limited range of peptides. To further evaluate the role of the hydroxyl group of the ZYA tyrosine, we replaced the hydroxyl with an amine or phosphate group. Both ZpYA and Z(nitro-Tyr]A failed to activate the 20S (Fig. 6D), indicating that hydrogen-bonding capacity in the penultimate position is important for $\mathrm{HbYX}$ function. This conclusion is further supported by the fact that Z(4-amino-Phe)A activates the 20S to a similar extent as ZYA (Fig. 6D). Unlike phosphotyrosine or 3-nitrotyrosine, 4-aminophenylalanine's mass and polar surface area is similar to tyrosine's, and it has the similar hydrogen bond availability.

\section{Comparing ZYA to known proteasome-activating small molecules}

Recently, several studies have been published on the potential of small molecules to activate the 20S proteasome through unclear mechanisms. We compared how ZYA, designed to mimic the HbYX motif, performs relative to some of these small molecules (AM-404, TCH-165, Fluspirilene (FLP) ${ }^{29,30}$ ). The other small molecules exhibited higher binding affinity relative to ZYA (with $\mathrm{K}_{\mathrm{obs}}$ values in the micromolar range). However, as indicated by $V_{\max }$ values, none of them activated the human $20 \mathrm{~S}$ to the extent that ZYA was capable of even at saturating concentrations (Table 1, SFig. 2). Interestingly, none of the other compounds significantly activated any forms of the T20S (WT, $a \Delta N, K 66 \mathrm{~A})$ (SFig. 3). It is expected that a gate-opening compound should not stimulate an open-channel 20S mutant, and that a HbYX mimetic should not activate the K66A mutant. Notably, ZYA activates WT T20S, whereas these other small molecules that are predicted to open the 20S gate, do not activate T20S at all. This suggests that ZYA structure still conserves the HbYX-dependent mechanism of gate opening since it activates proteasomes from archaea to mammals. The ability of a small molecule such as ZYA to activate the proteasome nearly 50 -fold is, to our knowledge, unprecedented.

\section{ZYA abrogates the proteasome-inhibiting activity of three different A11 + oligomers}

Thus far, we have demonstrated that ZYA increases peptide and protein degradation by inducing $20 \mathrm{~S}$ proteasome gate-opening. We recently published a study demonstrating that A11+oligomers (specific conformations of $A \beta$, $\alpha$-synuclein, and huntingtin with a $Q 53$ expansion) allosterically inhibit $20 S$ function by stabilizing the closed gate conformation ${ }^{20}$. Since ZYA stimulates gate opening, we investigated the possibility that it could also rescue the $20 \mathrm{~S}$ from inhibition by $\mathrm{A} 11+$ neurodegenerative-associated oligomers. Remarkably, when 20 S activity is measured in the presence of oligomers, the addition of 
$500 \mu \mathrm{M}$ ZYA completely blocks inhibition by $\mathrm{A} 11+$ oligomer (Fig. 7). Furthermore, at $50 \mu \mathrm{M}, \mathrm{ZYA}$ stimulates beyond the basal $20 S$ rates in the presence of oligomers (compare control with no ZYA to $A \beta \star 56$ at $50 \mu M$ ZYA). This is an unequivocal demonstration for the potential of small molecules to restore proteasome activity in conditions of 20S impairment. In addition, compounds that function like ZYA could also enhance degradation of IDPs (Fig. 5D\&E), which are often involved in neurodegenerative diseases (Fig. 8).

\section{Discussion}

NDs are characterized by progressive loss of neuronal functions and have been reported to exhibit abnormally low proteasomal activity, despite relatively normal amounts of cellular proteasomes ${ }^{2}$. In fact, for decades now, research has implicated some form of impairment of the proteasome system in the etiology of neurodegeneration diseases such as Alzheimer's and Parkinson's ${ }^{3-7}$. This is concomitant with the accumulation of intercellular proteins, many of which are intrinsically disordered proteins (IDPs) ${ }^{31-33}$. The function of the UPS is critical for the healthy maintenance of the proteome in general, and more specifically, for neuronal synapse function (i.e. plasticity and synaptic protein turnover) ${ }^{1,34}$.

The proteasome is a highly regulated multi-subunit complex and possesses multiple drug targets to modulate its degradation activity. The first compounds to target the proteasome inhibited the proteolytic sites of the $20 S^{35}$. More recently, compounds that inhibit a 19S-associated deubiquitinase (USP14) have also been developed ${ }^{36}$. Treatment of cultured cells with these deubiquitinase inhibitors can increase the rate of degradation of a subset of proteasome substrates (by increasing the substrate residence time on the proteasome $)^{36}$, and accelerate the rate of degradation of wild-type and pathological tau mutants ${ }^{37}$. Currently, the development of 20S gate-stimulating compounds aimed to restore proteostasis are beginning to receive attention, though the biological consequences of these compounds are unclear. Fortunately, a few studies have shown that genetically opening the 20S gate is tolerable to yeast and at least one mammalian cell line ${ }^{8,38}$, which supports the potential use of $20 \mathrm{~S}$ gate-stimulating compounds. Coleman et. al recently showed that treating HEK-293Ts with 20S-stimulating compounds of varying mechanisms of stimulation increased degradation of endogenous, primarily disordered, proteins ${ }^{39}$. The approach of stimulating the 20S may be beneficial to help restore proteostasis and prevent the accumulation of toxic aggregates by favoring proteasomal degradation of the monomeric forms of neurodegenerative-associated IDPs ${ }^{38}$.

Cryo-EM structures of the $26 \mathrm{~S}^{18,19,23,24}$ have suggested potential mechanisms for how the HbYX motif might induce gate-opening, consequently informing proteasome-stimulating strategies. However, details such as the minimum number of $\mathrm{HbYX}$-binding sites required or the exact $\mathrm{C}$-terminal tail that dictates gate-opening remains elusive. Structural analyses also do not precisely indicate the kinetics of the 20S gate when stimulated by the $\mathrm{HbYX}$ motif or the degree of openness of the gate as structure determination relies on averaging of large populations. Possibly, the increasing number of C-terminal binding affects the dynamics (fluctuations) of the $20 \mathrm{~S}$ gate but is overlooked due to the limitations of Cryo-EM, which is still 
an ensemble technique. This study is intended to expand our understanding of the HbYX-dependent gateopening mechanism and to ultimately inform the design of $20 \mathrm{~S}$ gate-opening small molecules.

By analyzing the $\mathrm{HbYX}$-motif interactions with the intersubunit pockets, we identify multiple residues critical for HbYX-dependent gate opening. Among the multiple crucial residues, our results indicate that L81's interaction with the tyrosine of the HbYX motif is primarily responsible for gate-opening, supported by the fact that mutation of this lysine to a tyrosine in the archaeal $20 \mathrm{~S}$ (presented here) stimulates gate opening (Fig. 2D). Furthermore, our results with this L81Y mutant combined with the ZYA tyrosine analogs (Fig. 6C) indicate that the tyrosine's extra density along with the added hydrogen bond with the backbone helix 0 G19 (Fig. 2E \& 6C) are important for maximal gate-opening. The conformational changes observed in our cryo-EM reconstruction of the L81Y mutant suggests that the interaction with the HbYX motif's tyrosine alone is sufficient to induce conformational changes in the gate and the a subunits.

Besides gate-opening, our reconstruction suggests that the L81Y mutation causes a pore expansion, as the Pro17s move away from the central pore. Comparison of WT and L81Y from the sides indicates the a subunits undergo a rocking motion that opens the gate (Fig. 3B, SMovie 1 \&2), which suggest that gateopening does not solely involve the gating regions, but rather the entire a subunit. These observations also rule out the functional necessity of (but not the existence of) other potential non-HbYX mechanism (e.g., activation loop) that could be involved in gate-opening. The minimal structural perturbation of the L81Y mutation indicates that the closed state of the gate is only minimally stabilized in both archaea and eukaryotic 20S, which allows for even small molecules (e.g., ZYA) to bind to this region near L81 in the intersubunit pocket and allosterically switch the state of the gate. Additionally, our observations suggests that other interactions in the a intersubunit pockets, such as contribution from the $\mathrm{Hb}$ residue may allosterically contribute to gate-opening (Fig. 2D; and likely increase C-terminal affinity) since the L81YT20S was not fully activated.

We hypothesize that the L81Y mutation would impose a similar effect in the eukaryotic 20S, if strategically targeted in the appropriate intersubunit pocket(s), as structures suggest that the intersubunit pockets in eukaryotic $20 \mathrm{~S}$ do not equally dictate gate-opening. In further support of this is the similarity of the conformational changes in the L81Y-T20S compared to the gate-opened form of the 26S proteasome (e.g. state $\left.E_{D 1 / D 2}\right)^{18,23}$, observed in a morph between the closed $\left(E_{A}\right)$ and open $\left(E_{D 1}\right)$ states of the $20 \mathrm{~S}$ in a $26 \mathrm{~S}$ structure (SMovie 4). Despite the expected asymmetry of conformational changes in the eukaryotic $20 \mathrm{~S}$, the radial-rotation of the alpha-subunits leading to $20 \mathrm{~S}$ pore expansion is remarkably similar to that seen in the L81Y-T20S structure (compare SMovie 1\&4). These conformational similarities leading to gate-opening suggests that the L81Y mutation recapitulates the conserved HbYX-dependent mechanism of gate-opening.

Deriving from the $\mathrm{HbYX}$ motif, our understanding of the intersubunit pocket, and iterative peptide design, we eventually formulated ZYA, a minimal mimetic of the motif that evidently satisfies interactions necessary for gate-opening. Based on our biochemical assays using constitutively open proteasomes 
(a $\Delta \mathrm{N}-\mathrm{T} 20 \mathrm{~S} \& \mathrm{a} 3 \Delta \mathrm{N}-\mathrm{Y} 20 \mathrm{~S}$ ), we can conclude that ZYA robustly induces $20 \mathrm{~S}$ gate opening (Fig. 3E \& 4B). In turn, we observe that ZYA stimulates both peptide hydrolysis and protein degradation (Fig. 4C \& D). Based on the cooperative binding of ZYA with a hill coefficient of 1.5, it is likely at least two ZYA molecules must be bound before gate-opening to occurs (Fig. 4A). This result is consistent with the suggestion that several $\mathrm{HbYX}$ motifs must have to bind to the intersubunit pockets to induce the necessary allosteric transitions to induce gate opening.

While our biochemical study of ZYA suggest the minimum number of HbYX bound to induce gateopening, it does not indicate which intersubunit pocket or pockets are most critical for gate-opening. The three primary HbYX motifs (Rpts 2, 3 \& 5) in the gate-closed 26S (e.g., state $E_{b}$ ) are bound to the 20S with an empty intersubunit between each of them. Only when the other C-termini (Rpt 1 and 6) begin to bind to these empty neighboring pockets is gate-opening observed. We speculate that $\mathrm{HbYX}$ binding to adjacent pockets may be the necessary trigger to switch the gate to open, which would be consistent with our results shown here and with several prior cryo-EM studies. In support of this hypothesis, Schnell et. al recent findings showed that $\mathrm{Pba} 1 / 2$, chaperones possessing $\mathrm{HbYX}$ motifs, bound to neighboring intersubunit pockets (a5-6 and a6-7) and induced gate opening, allowing the entry of an inhibitory Ndomain. They also showed that removing the inhibitory $\mathrm{N}$-domain allowed the complex $\left(\mathrm{Pba}^{\Delta 12} / 2\right)$ to activate $20 \mathrm{~S}$ peptide degradation ${ }^{40}$. Therefore, based on our study and those discussed above ${ }^{18,19,23,24}$, it is likely that the HbYX motif needs to bind to two neighboring intersubunit pockets to effectively induce gate opening. Furthermore, it seems reasonable to suggest that most critical intersubunit pockets to be occupied by the HbYX motif would likely be those that affect the N-temini of a2, a3 and a4, since these are the most critical for gating. Although, as part of an allosteric system, it's difficult to predict which two pockets would be most effective. Further structural studies will be required to elucidate the exact intersubunit pocket(s) that dictate gate-opening in the $26 \mathrm{~S}$ proteasome.

Does ZYA function as a minimal HbYX motif? First, ZYA failed to stimulate T20S with a K66A mutation (Fig. 3D), suggesting that ZYA interacts with the intersubunit pocket similarly to the HbYX motif (Fig. 2C). Additionally, ZYA's $\mathrm{Hb}$ group, penultimate tyrosine and the C-terminal carboxy of ZYA were all critical for the induction of gate opening (Fig. 6). Collectively, these results indicate that ZYA does function as a minimal motif for inducing gate-opening in the archaeal and eukaryotic 20S proteasome. ZYA should serve well as a research tool to specifically induce $\mathrm{HbYX}$-dependent gate-opening, based on its specificity and robust activity (e.g., it works far better than SDS or any other compound (Table 1) we've found to induce gate-opening). ZYA's low affinity ( $\mathrm{k}_{\mathrm{obs}} \approx 1 \mathrm{mM}$, Fig. $4 \mathrm{~A}$ ) in comparison to PAs with HbYX motifs is likely due to various factors. The dipeptide has a relatively large number of rotatable bonds and thus a high degree of freedom structurally, which can limit on-rates and reduce affinity. The relatively higher affinity of PAs also comes from their use of several C-termini and domains to associate with the 20S. Regardless of the type of PA ( $\mathrm{HbYX}$-dependent or -independent), there appears to be a common theme of multivalent binding and allostery for the gate to stably open (PA200/BIm10 is the exception here as it only has a single HbYX motif but manages to induce gate-opening as well). The structure of PA26-20S suggest that $11 \mathrm{~S}$ activators interface with the a ring at seven contact points ${ }^{9} .19 S-20$ S structures ${ }^{41}$ also 
indicate multiple contact points similarly using the C-terminal tails of the base subcomplex to dock in the a intersubunit pockets. Thus, avidity contributes to PAs high affinity, which are not realized for a small peptide like ZYA. ZYA's low affinity, in addition to its cooperative binding, suggests multivalent binding and allostery are significant components of $20 \mathrm{~S}$ gate-opening mechanisms.

Here, we showed that a simple replacement of L81 in the T20S with a tyrosine, or binding of ZYA, both appear to emulate the binding of the HbYX motif to the a intersubunit pockets resulting in gate opening. Previously, we showed that this HbYX-dependent mechanism is allosterically connected to the mechanism of impairment by ND-related A11+ oligomers ${ }^{20}$. Our results here showed that even at concentrations much less than saturating, ZYA is effective at reversing proteasome inhibition by three different A11+ ND-related proteins (Fig. 7). We expect compounds that could similarly induce gate opening at more physiologically relevant affinities could potentially treat ND. Such compounds could both stimulate degradation of most ND-related proteins, which are typically IDPs, and simultaneously reverse proteasome impairment, which has been observed in aging (Fig. 8). Advancements in understanding the connection between these two contrary $20 \mathrm{~S}$ gate-regulatory mechanisms provide a framework for the development of small molecules that antagonize proteasome impairment, especially by $\mathrm{A} 11+$ oligomers, or activate its ability to degrade unstructured proteins. Considering the importance of the proteasomal gate in regulating protein degradation, gate-activating compounds (such as ZYA) have promising potential as research tools to probe proteasome function in vitro and in vivo, and perhaps, elucidate the role of proteasome impairment in neurodegenerative disease progression.

\section{Methods}

\section{Proteasome purifications}

T.acidophilum wild type (WT) 20S and $\Delta \mathrm{a} 2-1220$ S proteasomes were purified as described. All $20 \mathrm{~S}$ mutants were generated by overlapping PCR site-directed mutagenesis. The plasmid for M.jannaschii PAN(M74A), kindly provided by Dr. Peter Zwickl30, lacked a His tag and was purified as described38, but Tris buffers were made at $50 \mathrm{mM}$ instead of $20 \mathrm{mM}$. Mammalian $20 \mathrm{~S}$ proteasomes were isolated from bovine liver as described39. WT and mutant a3 $\Delta N$ yeast $20 \mathrm{~S}$ proteasomes were expressed and purified by anion-exchange chromatography as de-scribed40. Human proteasomes, tagged with HTBH on $\beta 4$ subunits, were affinity purified from HEK293T cells as described ${ }^{42}$ with slight modifications. Cells were resuspended in Lysis Buffer (50mM Tris, protease inhibitors) and sonicated. Lysates were centrifuged at $20,000 \times \mathrm{g}$ and supernatants were incubated with streptavidin agarose resin (Millipore) for $1 \mathrm{~h}$ at RT. The beads were then incubated with biotin tagged-TEV protease in $50 \mathrm{mM}$ Tris overnight at RT.

\section{Proteasome activity assays - peptide substrates}

Fluorogenic substrate peptides were purchased from BostonBiochem (suc-LLVY-amc) and EZBiolabs (acnLPnLD-amc, ac-RLR-amc, LFP (Mca-AKVYPYPME-Dpa(Dnp)-am-ide)), PAN CT peptides, ZYA, and ZYA derivatives were synthesized by ABclonal. Compounds (AM-404, TCH-165, Fluspirilene (FLP)) were 
purchased from Cayman Chemical. Peptides and chemical compounds were dissolved in DMSO and incubated with proteasomes at indicated concentrations. The final concentration of DMSO in activity assays was $2 \%$. Oligomers of $A \beta \star 56$, $a$-synuclein, and huntingtin-Q53 were prepared as described 41 . All oligomers used were recognized by the a-oligomer antibody, A1142. Protein concentrations were determined by Bradford assay (Thermo Scientific). To measure peptide hydrolysis, fluorogenic peptides dissolved in DMSO were used at a final concentration of 25-100 $\mu \mathrm{M}$ for Suc-LLVY-amc and Ac-nLPnLDamc, and 3-10 $\mu \mathrm{M}$ for LFP and Boc-RLR-amc, in $50 \mathrm{mM}$ Tris (pH 7.5), $1 \mathrm{mM}$ DTT. For archaeal $20 \mathrm{~S}$ experiments the indicated concentrations of T20S and LFP peptide was added to the buffer at $45^{\circ} \mathrm{C}$, and where indicated, $1 \mu \mathrm{g}$ of PAN and $10 \mu \mathrm{M}$ ATPYS (+5 mM MgCl2) was added to the $0.1 \mathrm{ml}$ of reaction buffer (sufficient to saturate the 20S particles)43. Assays with mammalian (0.5 nM and $1 \mathrm{nM}$ as indicated), yeast wild-type $(2 \mathrm{nM})$, and yeast a $3 \Delta \mathrm{N}(0.2 \mathrm{nM})$ proteasomes were performed at $37^{\circ} \mathrm{C}$. Assays with $A \beta$, a-synuclein, and huntingtin-Q53 oligomers were performed as described ${ }^{20}$.

\section{Proteasome activity assays - protein substrates}

Tau23 (gift from Eckhard Mandelkow) or $\beta$-casein (Sigma) were incubated with mammalian 20S proteasomes for the indicated time at $37^{\circ} \mathrm{C}$. Reactions were quenched by addition of LDS sample buffer (Invitrogen). Proteins were separated by SDS-PAGE using NuPAGE ${ }^{\mathrm{TM}} 4-12 \%$ Bis-Tris protein gels (Invitrogen) and visualized with Coomassie brilliant blue. Degradation of ${ }^{14} \mathrm{C}$-Casein was assessed by scintillation of acid soluble counts as described in ${ }^{43}$.

\section{Cryo-EM Sample Preparation and Data Collection}

Copper Quantifoil R 1.2/1.3 300 mesh (EMS) grids were cleaned using a PELCO easiGlow Glow Discharge cleaning system. A volume of $3 \mathrm{uL}$ of $0.5 \mathrm{mg} / \mathrm{mL}$ L81Y T20S sample was placed onto a grid, and then flash frozen in liquid ethane using a manual plunge freeze apparatus. Data collection was done using a Titan Krios transmission electron microscope (Thermo Fisher) operated at $300 \mathrm{~kW}$ and a magnification of $\mathrm{x} 81,000$, which resulted in $0.503 \AA$ px. Images were collected using a Falcon IIIEC direct electron detector camera equipped with a $\mathrm{K} 3 / \mathrm{GIF}$ operating in counting and super resolution modes. Electron dose per pixel of $50 \mathrm{e}-/ \AA 22$ was saved as 40 frame movies within a target defocus range of -2.5 to -1.25 . All the data was collected using cryoSPARC software (Thermo Fisher) ${ }^{44}$.

\section{Cryo-EM Single Particle Analysis}

Cryo-EM images of the WT and L81Y T20S proteasome were analyzed using cryoSPARC. L81Y T20S: From the 2850 movies collected, we used 2847 in analysis and picked 889,069 particles after three rounds of 2D classification to obtain the best particle sets. The particles chosen from 2D classification were used to generate an Ab-initio model, which was used for homogeneous refinement. Final map was imported into Phenix ${ }^{45}$ to run density modification (DenMod) from two half maps. All representations (figures and movies) of the T20S proteasome complex were created using UCSF ChimeraX ${ }^{46,47}$.

\section{Computational Docking}


Automated docking of the ZYA peptide mimetic was done with Glide from the Schrodinger Suite ${ }^{48}$

\section{Statistical analysis}

Data were analyzed in Graph Pad or excel using an unpaired Student's t-test (Prism). For all statistical analyses, a value of $p<0.05$ was considered significant.

\section{Data availability}

The authors declare that data supporting the findings of this study are available within the paper and its supplementary information files and are available from the corresponding author upon request.

\section{Declarations}

\section{ACKNOWLEDGEMENTS}

We thank the members of the Smith Lab for the helpful and valuable discussions. We thank our undergraduates, Matthew Dombrowski and Matthew Rexroad, for their assistance. We thank Dr. Andrew K. Shiemke for careful and meticulous review of our manuscript. Transmission electron micrographs were recorded at the University of Virginia Molecular Electron Microscopy Core facility (RRID:SCR_019031), which is supported in part by the School of Medicine and built with NIH grant G20-RR31199. In addition, the Titan Krios (S10-RR025067), Falcon II/3EC direct detector (S10-OD018149), and K3/GIF (U24GM116790) were purchased in part or in full using the designated NIH grants. Molecular graphics and analyses performed with UCSF ChimeraX, developed by the Resource for Biocomputing, Visualization, and Informatics at the University of California, San Francisco, with support from National Institutes of Health R01-GM129325 and the Office of Cyber Infrastructure and Computational Biology, National Institute of Allergy and Infectious Diseases. This work was supported by NIH-R01AG064188 to D.M.S.

\section{AUTHOR CONTRIBUTIONS}

J.J.C. performed experiments using acetylated peptides, ZYA, WT, K66A, and a $\Delta N$ T20S, WT and a3 $\Delta N$ Y20S. T.A.T. performed ZYA dose response, and tested ZYA analogs and oligomers experiments. D.M.S. performed protein degradation assays. J.J.C. purified WT, a $\Delta N$, K66A T20S and WT and a3 $\Delta N$ Y20S, and mammalian 20S. T.A.T. purified mammalian 20S, and prepared amyloid- $\beta$, a-synuclein, and huntingtin oligomers. D.M.S. purified K33G, L81A, L81G, L81Y, K66A T20S and ${ }^{14} \mathrm{C}$ casein. T.A.T. performed the docking of ZYA in the human proteasome. J.J.C. purified L81Y T20S for cryo-EM. J.J.C and D.M.S. analyzed cryo-EM data, reconstructed the L81Y proteasome structure, and generated supplemental movies. Results were analyzed and interpreted by J.J.C., T.A.T., and D.M.S. Manuscript was prepared by J.J.C., T.A.T., and D.M.S. All authors reviewed the results and approved the final version of this manuscript. 


\section{COMPETING INTERESTS}

The authors declare no competing interests.

\section{References}

1. Thibaudeau, T. A. \& Smith, D. M. A Practical Review of Proteasome Pharmacology. Pharmacol. Rev. 71, 170-197 (2019).

2. Keller, J. N., Hanni, K. B. \& Markesbery, W. R. Impaired Proteasome Function in Alzheimer's Disease. J. Neurochem. 75, 436-439 (2000).

3. Ciechanover, A. \& Brundin, P. The ubiquitin proteasome system in neurodegenerative diseases: sometimes the chicken, sometimes the egg. Neuron 40, 427-446 (2003).

4. McNaught, K. S., Olanow, C. W., Halliwell, B., Isacson, O. \& Jenner, P. Failure of the ubiquitinproteasome system in Parkinson's disease. Nat. Rev. Neurosci. 2, 589-594 (2001).

5. Ortega, Z., Díaz-Hernández, M. \& Lucas, J. J. Is the ubiquitin-proteasome system impaired in Huntington's disease? Cell. Mol. Life Sci. CMLS 64, 2245-2257 (2007).

6. McKinnon, C. \& Tabrizi, S. J. The ubiquitin-proteasome system in neurodegeneration. Antioxid. Redox Signal. 21, 2302-2321 (2014).

7. McNaught, K. S. P. et al. Proteasome inhibition causes nigral degeneration with inclusion bodies in rats. Neuroreport 13, 1437-1441 (2002).

8. Groll, M. et al. A gated channel into the proteasome core particle. Nat. Struct. Biol. 7, 1062-1067 (2000).

9. Förster, A., Masters, E. I., Whitby, F. G., Robinson, H. \& Hill, C. P. The 1.9 A structure of a proteasome$11 \mathrm{~S}$ activator complex and implications for proteasome-PAN/PA700 interactions. Mol. Cell 18, 589599 (2005).

10. Religa, T. L., Sprangers, R. \& Kay, L. E. Dynamic regulation of archaeal proteasome gate opening as studied by TROSY NMR. Science 328, 98-102 (2010).

11. Ruschak, A. M. \& Kay, L. E. Proteasome allostery as a population shift between interchanging conformers. Proc. Natl. Acad. Sci. U. S. A. 109, E3454-3462 (2012).

12. Köhler, A. et al. The axial channel of the proteasome core particle is gated by the Rpt2 ATPase and controls both substrate entry and product release. Mol. Cell 7, 1143-1152 (2001).

13. Finley, D., Chen, X. \& Walters, K. J. Gates, Channels, and Switches: Elements of the Proteasome Machine. Trends Biochem. Sci. 41, 77-93 (2016).

14. Stadtmueller, B. M. \& Hill, C. P. Proteasome activators. Mol. Cell 41, 8-19 (2011).

15. Rabl, J. et al. Mechanism of gate opening in the 20 S proteasome by the proteasomal ATPases. Mol. Cell 30, 360-368 (2008).

16. Smith, D. M. et al. Docking of the Proteasomal ATPases' C-termini in the 20S Proteasomes alpha Ring Opens the Gate for Substrate Entry. Mol. Cel/ 27, 731-744 (2007). 
17. Dange, T. et al. Blm10 Protein Promotes Proteasomal Substrate Turnover by an Active Gating Mechanism. J. Biol. Chem. 286, 42830-42839 (2011).

18. Dong, Y. et al. Cryo-EM structures and dynamics of substrate-engaged human $26 \mathrm{~S}$ proteasome. Nature 565, 49-55 (2019).

19. Eisele, M. R. et al. Expanded Coverage of the 26S Proteasome Conformational Landscape Reveals Mechanisms of Peptidase Gating. Cell Rep. 24, 1301-1315.e5 (2018).

20. Thibaudeau, T. A., Anderson, R. T. \& Smith, D. M. A common mechanism of proteasome impairment by neurodegenerative disease-associated oligomers. Nat. Commun. 9, 1097 (2018).

21. Whitby, F. G. et al. Structural basis for the activation of $20 \mathrm{~S}$ proteasomes by $11 \mathrm{~S}$ regulators. Nature $408,115-120(2000)$.

22. Förster, A., Whitby, F. G. \& Hill, C. P. The pore of activated 20 S proteasomes has an ordered 7-fold symmetric conformation. EMBO J. 22, 4356-4364 (2003).

23. Peña, A. H. de la, Goodall, E. A., Gates, S. N., Lander, G. C. \& Martin, A. Substrate-engaged $26 \mathrm{~S}$ proteasome structures reveal mechanisms for ATP-hydrolysis-driven translocation. Science 362, (2018).

24. Wehmer, M. et al. Structural insights into the functional cycle of the ATPase module of the $26 \mathrm{~S}$ proteasome. Proc. Natl. Acad. Sci. U. S. A. 114, 1305-1310 (2017).

25. Kayed, R. et al. Common Structure of Soluble Amyloid Oligomers Implies Common Mechanism of Pathogenesis. Science 300, 486-489 (2003).

26. Groll, M., Brandstetter, H., Bartunik, H., Bourenkow, G. \& Huber, R. Investigations on the maturation and regulation of archaebacterial proteasomes. J. Mol. Biol. 327, 75-83 (2003).

27. Stadtmueller, B. M. et al. Structural models for interactions between the $20 \mathrm{~S}$ proteasome and its PAN/19S activators. J. Biol. Chem. 285, 13-17 (2010).

28. Campbell, M. G., Veesler, D., Cheng, A., Potter, C. S. \& Carragher, B. 2.8 Å resolution reconstruction of the Thermoplasma acidophilum $20 \mathrm{~S}$ proteasome using cryo-electron microscopy. eL ife 4, e06380 (2015).

29. Njomen, E. \& Tepe, J. J. Proteasome Activation as a New Therapeutic Approach To Target Proteotoxic Disorders. J. Med. Chem. 62, 6469-6481 (2019).

30. Jones, C. L. \& Tepe, J. J. Proteasome Activation to Combat Proteotoxicity. Mol. Basel Switz. 24, (2019).

31. Uversky, V. N. Intrinsically disordered proteins and their (disordered) proteomes in neurodegenerative disorders. Front. Aging Neurosci. 7, (2015).

32. Ross, C. A. \& Poirier, M. A. Protein aggregation and neurodegenerative disease. Nat. Med. 10, S10S17 (2004).

33. Walker, L. C. \& LeVine, H. Corruption and Spread of Pathogenic Proteins in Neurodegenerative Diseases*,. J. Biol. Chem. 287, 33109-33115 (2012). 
34. Lue, L. F. et al. Soluble amyloid beta peptide concentration as a predictor of synaptic change in Alzheimer's disease. Am. J. Pathol. 155, 853-862 (1999).

35. Rock, K. L. et al. Inhibitors of the proteasome block the degradation of most cell proteins and the generation of peptides presented on MHC class I molecules. Cell 78, 761-771 (1994).

36. Lee, B.-H. et al. Enhancement of proteasome activity by a small-molecule inhibitor of USP14. Nature 467, 179-184 (2010).

37. Boselli, M. et al. An inhibitor of the proteasomal deubiquitinating enzyme USP14 induces tau elimination in cultured neurons. J. Biol. Chem. 292, 19209-19225 (2017).

38. Choi, W. H. et al. Open-gate mutants of the mammalian proteasome show enhanced ubiquitinconjugate degradation. Nat. Commun. 7, 10963 (2016).

39. Coleman, R. A., Mohallem, R., Aryal, U. K. \& Trader, D. J. Protein degradation profile reveals dynamic nature of 20S proteasome small molecule stimulation. RSC Chem. Biol. 2, 636-644 (2021).

40. Schnell, H. M. et al. Structures of chaperone-associated assembly intermediates reveal coordinated mechanisms of proteasome biogenesis. Nat. Struct. Mol. Biol. 28, 418-425 (2021).

41. Schweitzer, A. et al. Structure of the human $26 \mathrm{~S}$ proteasome at a resolution of $3.9 \AA$. Proc. Natl. Acad. Sci. 113, 7816-7821 (2016).

42. Han, D. H. et al. Direct cellular delivery of human proteasomes to delay tau aggregation. Nat. Commun. 5, 5633 (2014).

43. Smith, D. M. et al. ATP binding to PAN or the 26S ATPases causes association with the $20 \mathrm{~S}$ proteasome, gate opening, and translocation of unfolded proteins. Mol. Cell 20, 687-698 (2005).

44. cryoSPARC: algorithms for rapid unsupervised cryo-EM structure determination I Nature Methods. https://www.nature.com/articles/nmeth.4169.

45. Liebschner, D. et al. Macromolecular structure determination using X-rays, neutrons and electrons: recent developments in Phenix. Acta Crystallogr. Sect. Struct. Biol. 75, 861-877 (2019).

46. Goddard, T. D. et al. UCSF ChimeraX: Meeting modern challenges in visualization and analysis. Protein Sci. Publ. Protein Soc. 27, 14-25 (2018).

47. Pettersen, E. F. et al. UCSF ChimeraX: Structure visualization for researchers, educators, and developers. Protein Sci. Publ. Protein Soc. 30, 70-82 (2021).

48. Friesner, R. A. et al. Glide: A New Approach for Rapid, Accurate Docking and Scoring. 1. Method and Assessment of Docking Accuracy. J. Med. Chem. 47, 1739-1749 (2004).

\section{Table}




\begin{tabular}{|l|l|l|l|}
\hline & $\mathbf{V}_{\max }$ (fold) & $\mathbf{K}_{\text {obs }}$ (uM) & $\mathbf{V}_{\max }(95 \% \mathrm{Cl})$ \\
\hline AM-404 & 12.86 & 12.06 & 10.23 to 17.37 \\
\hline TCH-165 & 2.12 & 0.83 & 1.722 to 2.568 \\
\hline FLP & 6.93 & 2.66 & 4.931 to 10.01 \\
\hline ZYA & 48.49 & 970 & 44.32 to 55.75 \\
\hline
\end{tabular}

Table 1. Comparison of proteasome stimulation ability and affinity of ZYA and other known compounds that activate proteasome function. Values derived from saturation curves in Supplemental Figure 2.

\section{Figures}
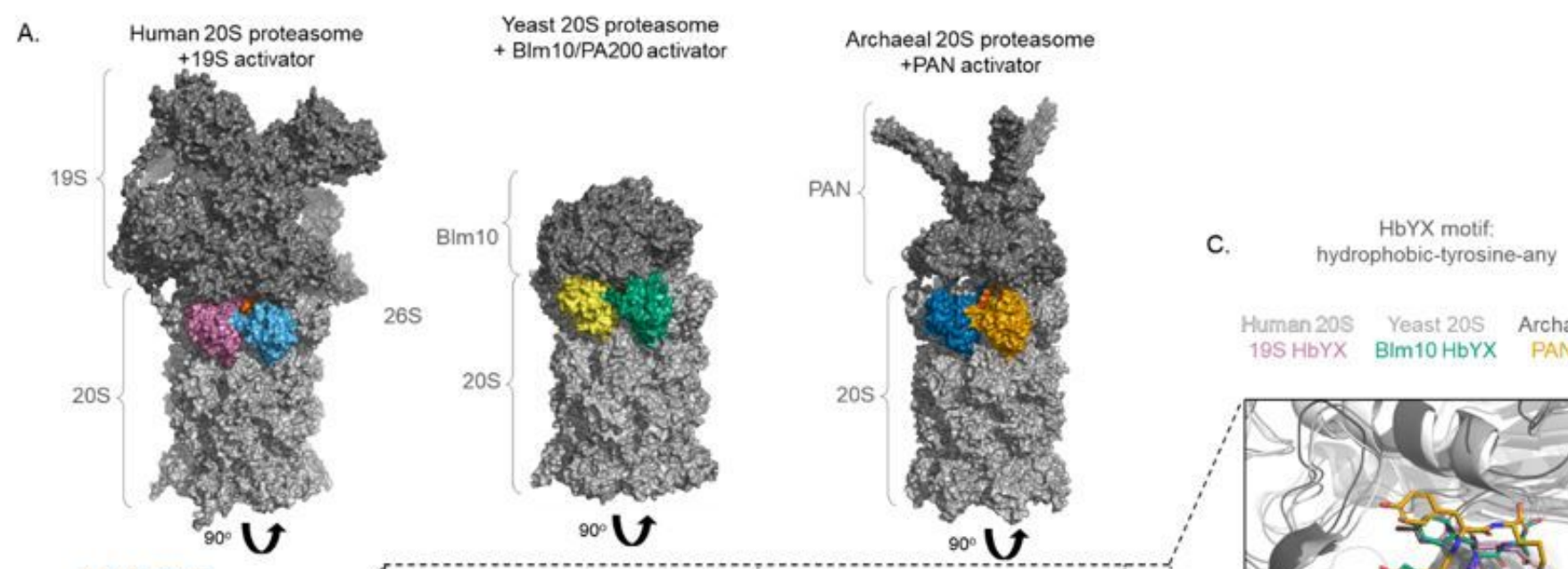

B.
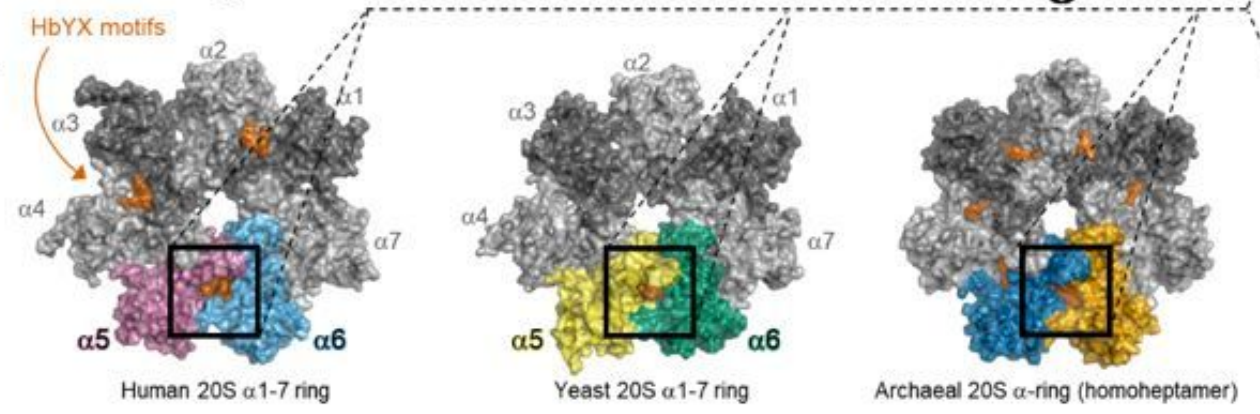

Human20S Yeast20S Archaeal20S $19 \mathrm{SHbYX}$ BIm10 HbYX PANHbYX

\section{Figure 1}

$\mathrm{HbYX}$ motifs from various proteasome activators docked in the $20 \mathrm{~S}$ a ring. A. Surface representation of $20 S$ proteasomes in complex with activators [Human 26S (PDB 6msk), yeast 20S+BIm10 (PDB 4v7o), archaeal 20S + PAN (PDB 6hed)]. HbYX motifs visible are colored redish-orange and adjacent a-subunits of the visible $\mathrm{HbYX}$ motif are shown in various colors. B. Surface representation of the $20 \mathrm{~S}$ a rings from $\mathrm{A}$ but rotated to show the 20S top view with activator caps removed. Proteasome activator C-termini HbYX residues are shown in redish-orange (surface). C. Overlay of $20 \mathrm{~S}$ intersubunit pockets (cartoon) from $B$ with indicated HbYX motif residues (sticks). Crystal structure of PAN C-terminus (PDB 3ipm) is shown in place of cryo-EM PDB 6hed. Images were rendered with PyMOL. 
A.

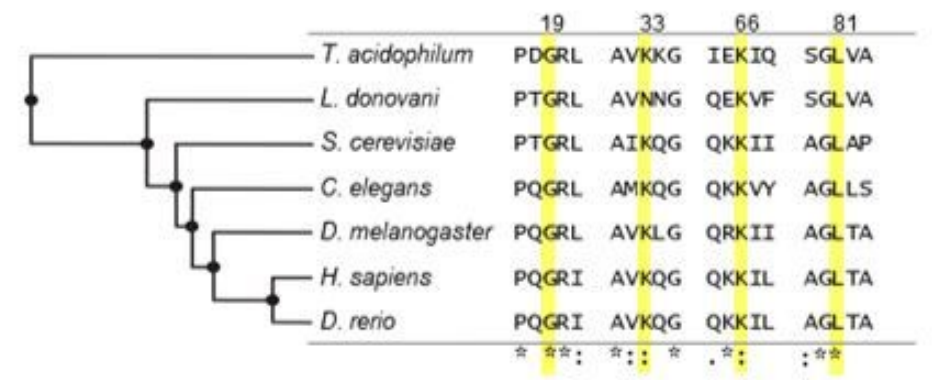

C.

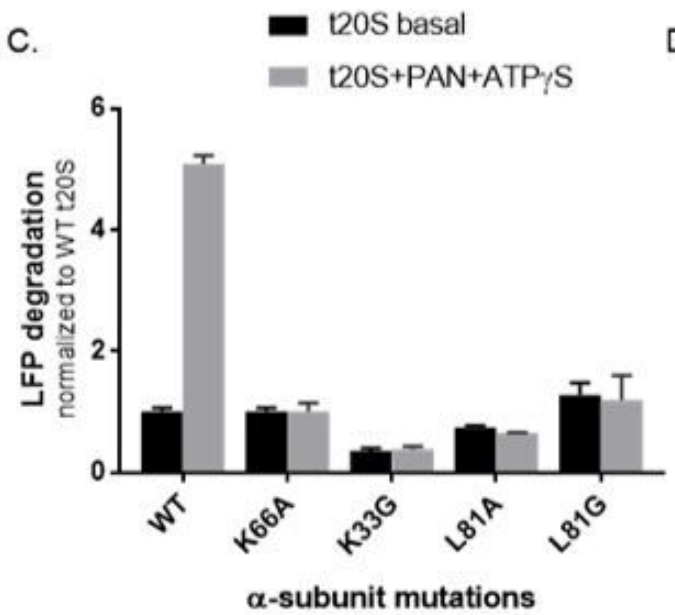

D.
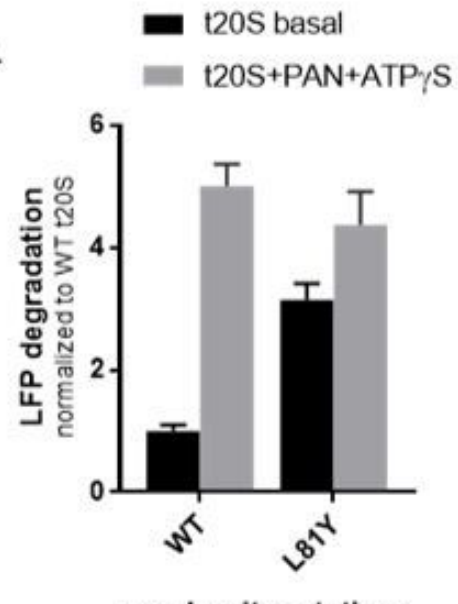

B.

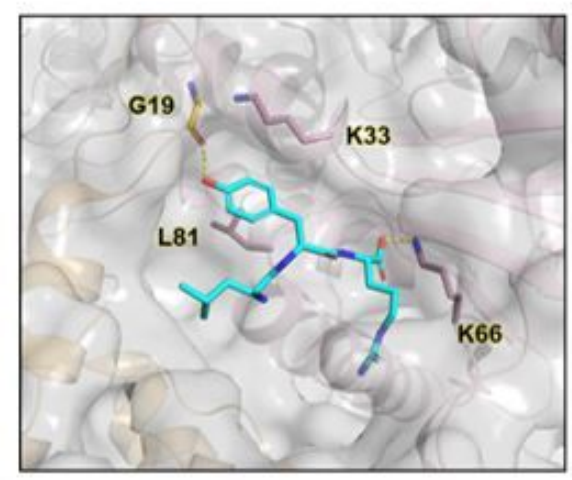

E.

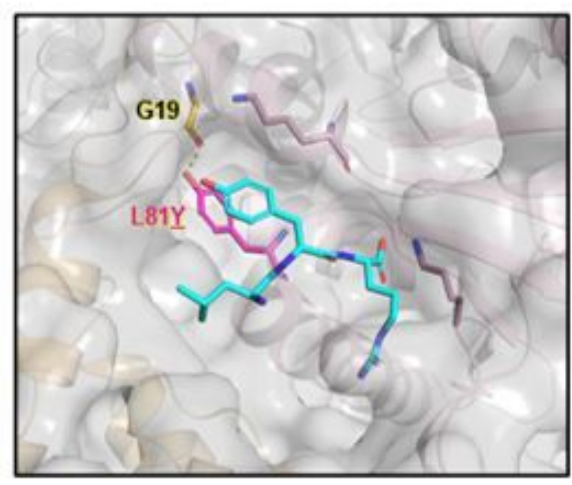

Figure 2

Conserved T20S a-intersubunit pocket residues are required for $\mathrm{HbYX}$-dependent gate opening. A. Multiple sequences alignment of $20 \mathrm{~S}$ a 6 protein generated with CLUSTAL OMEGA (1.2.4). B. Conserved residues interacting with bound HbYX motif (sticks) in the T20S intersubunit pocket (PDB 3ipm). PAN HbYX motif (LYR) shown in cyan (stick). C. Effect of PAN mutations on proteasome activity. T20S proteasome $(0.2 \mu \mathrm{g} / \mathrm{rxn}$ of wild-type (WT) or K66/K33/L81 mutants and fluorogenic nonapeptide (LFP) incubated with or without PAN and ATPYS. Stimulation of gate opening was measured by the increase of LFP hydrolysis (rfu/min) relative to WT 20S without PAN. D. Effect of 20S proteasome mutations on activity. Experiments with T20S proteasome (wild-type or L81Y mutant) performed as in C. E. same as (B), with L81 mutated to tyrosine (magenta stick). Images in B and E were rendered with PyMOL. Data are representative of the mean of three or more independent experiments performed in triplicate. Error bars represent \pm SD. 
A.

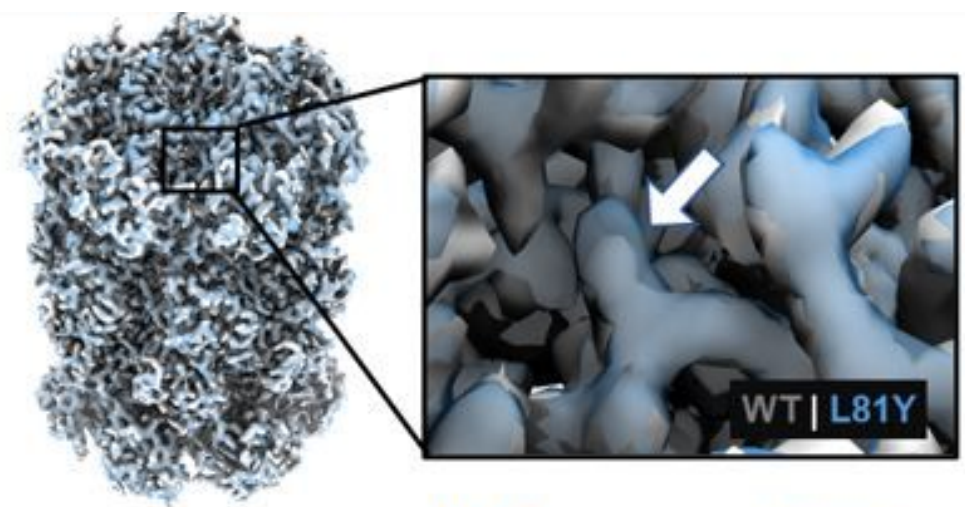

B.
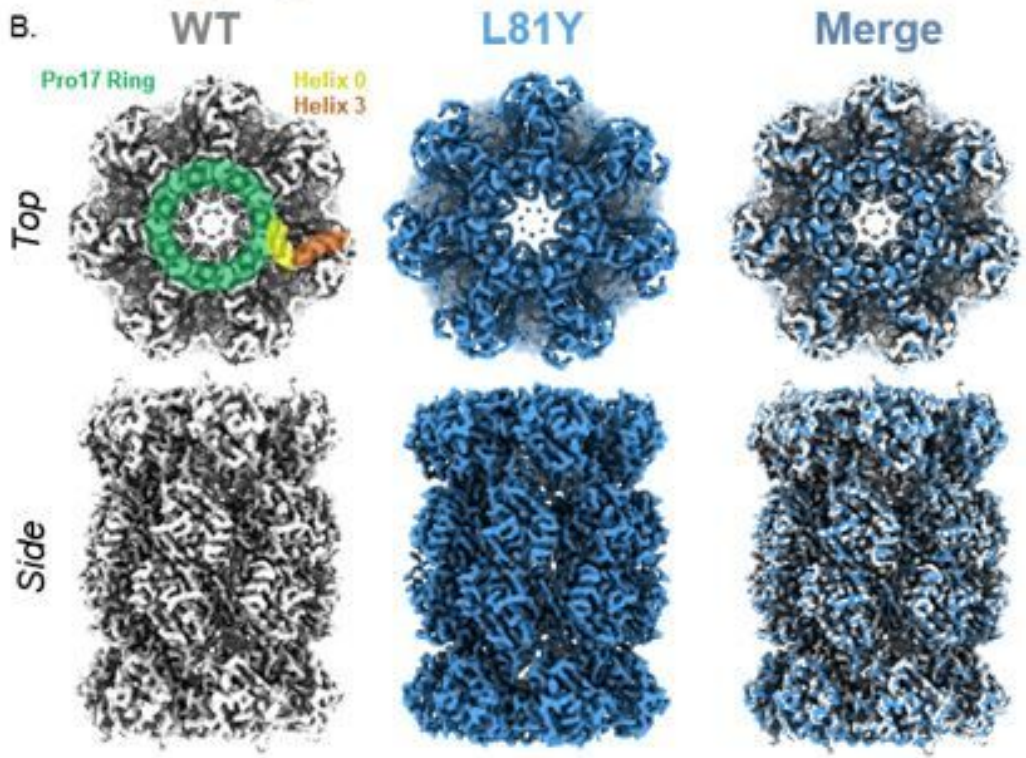

c.
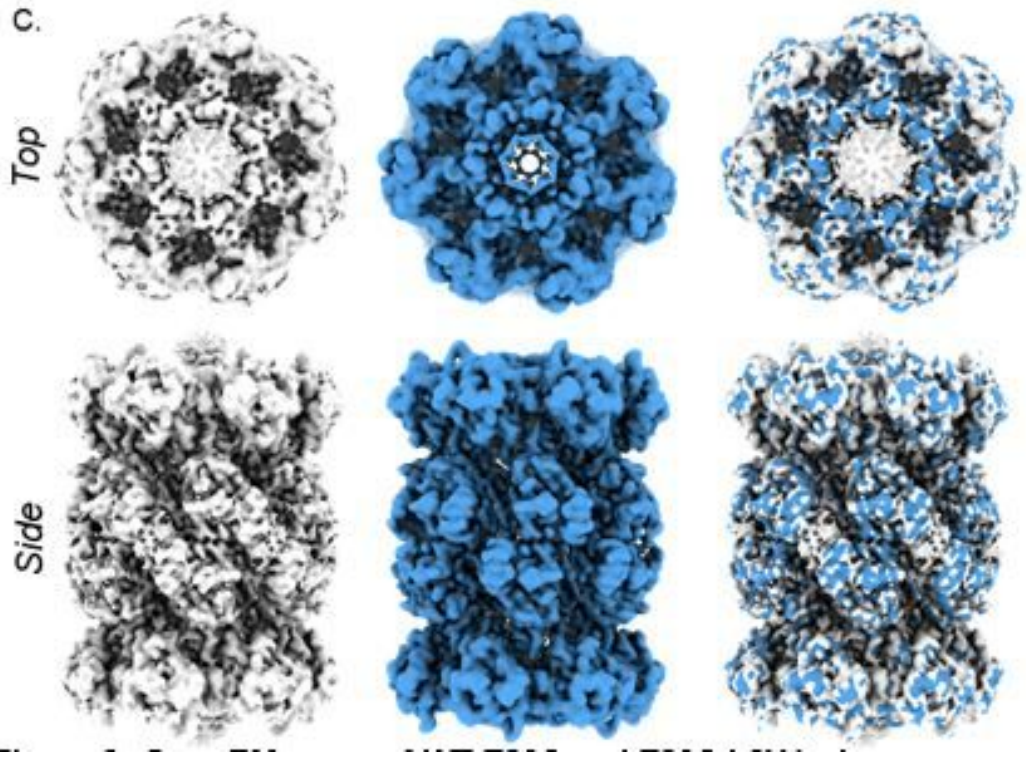

\section{Figure 3}

Cryo EM maps of WT T20S and T20S-L8Y1 show conformational changes induced by L81Y mutation that induce gate-opening. A. Superimposition of WT (gray) and L81Y (blue, transparent surface) EM maps. Box: density corresponding to L81 is larger in the map of L81Y T20S (blue) than WT T20S (gray) B. Top and side views of the 2.6Å EM Map of WT T20S (light gray), 2.0Å EM Map of L81Y T20S (blue), 
both maps merged (blue and white) showing conformational differences. C. Same as B but unsharpened maps.

A.

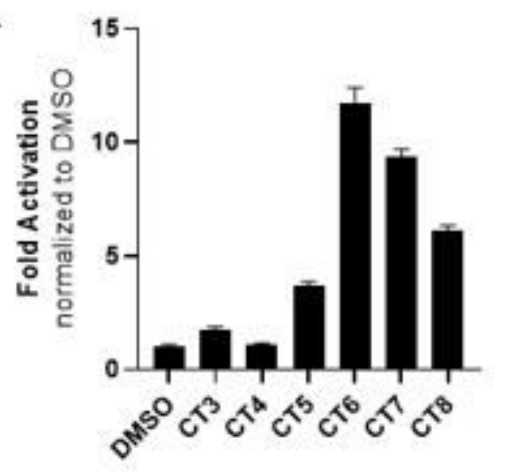

C.<smiles>CC(C)C(=O)NC(Cc1ccc(O)cc1)C(=O)NC(=O)OCc1ccccc1</smiles>

ZYA (Carboxybenzyl-Tyrosine-Alanine)
PAN C-terminus acetylated peptides

CTB AC-AHLDVLYR

CT7 AC-HLDVLYR

CT6 AC-LDVLYR

CT5 AC-DVLYR

CT4 AC-VLYR

CT3 AC-LYR

D.

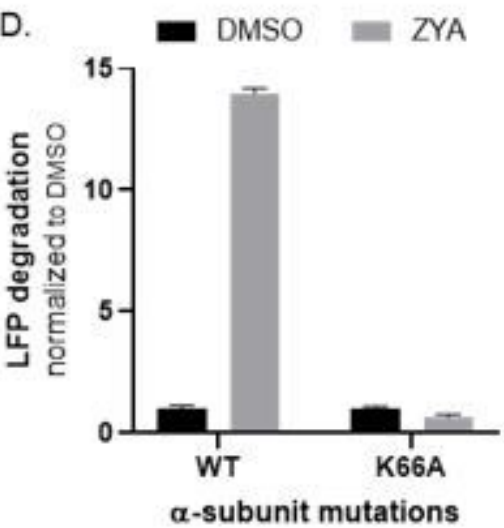

B.

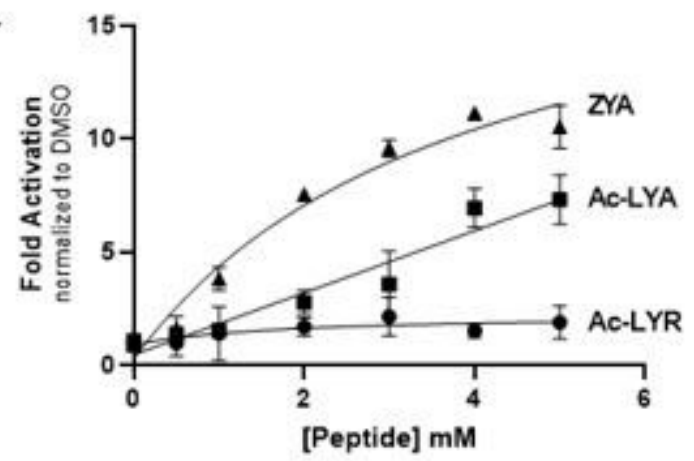

E.

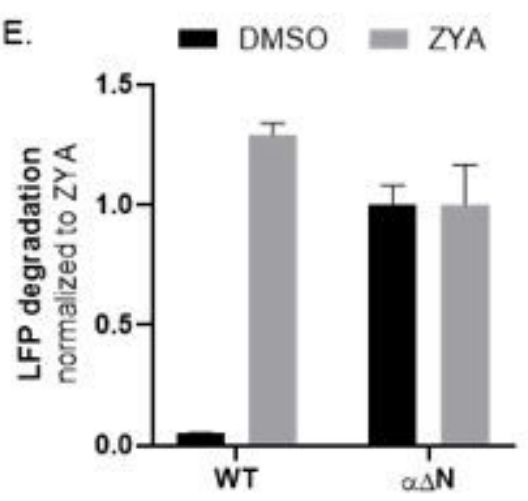

Figure 4

ZYA, a dipeptide mimetic of $\mathrm{HbYX}$, induces gate-opening in archaeal proteasomes (T20S) A. (Left) PAN Cterminal peptides $(200 \mathrm{mM})$ were incubated with wild type (WT) T20S $(0.5 \mu \mathrm{g} / \mathrm{rxn})$ and LFP. Stimulation of gate opening was measured by increase of LFP hydrolysis ( $\mathrm{rfu} / \mathrm{min}$ ) relative to WT T20S with DMSO.

(Right) sequences of peptides. B. Dose response activation of T20S by tripeptides (Ac-LYR, AC-LYA, ZYA) with wild-type T20S $(0.5 \mu \mathrm{g} / \mathrm{rxn})$ and LFP. LFP degradation rate $(\mathrm{rfu} / \mathrm{min})$ was normalized to DMSO. C. Structure of ZYA peptide mimetic. D. 2.5mM ZYA incubated with wild type (WT) or K66A T20S and LFP. LFP degradation rate (rfu/min) normalized to DMSO. E. 2.5mM ZYA incubated with wild type (WT) or gateless $(a \Delta N)$ T20S and LFP. LFP degradation rate (rfu/min) normalized to ZYA. Data are representative 
A.

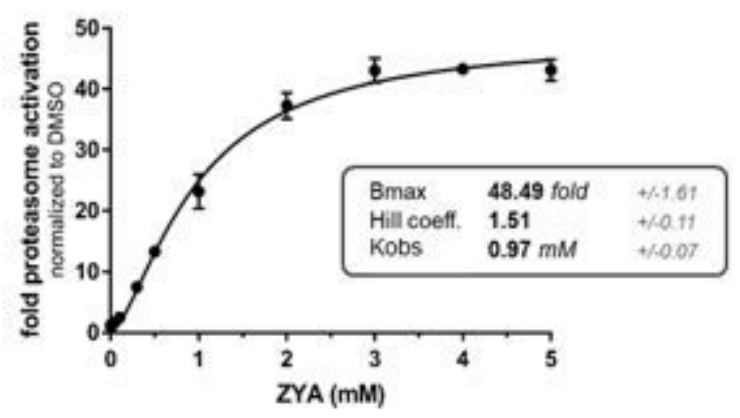

B.

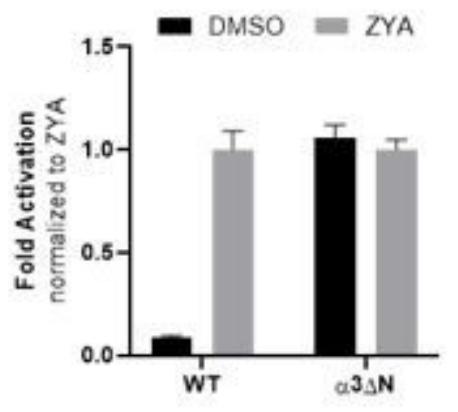

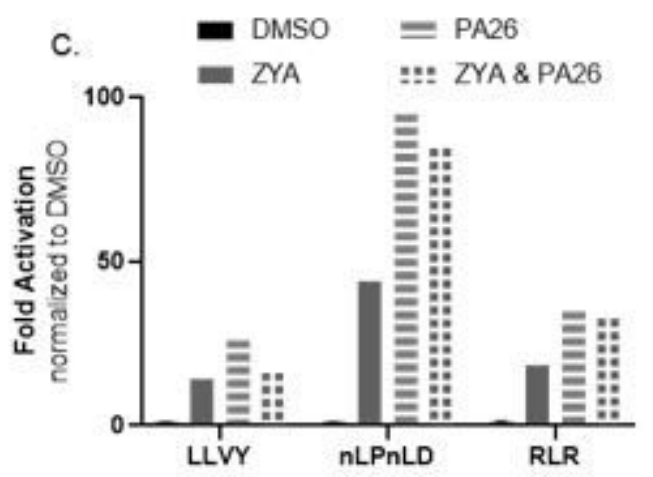

D.

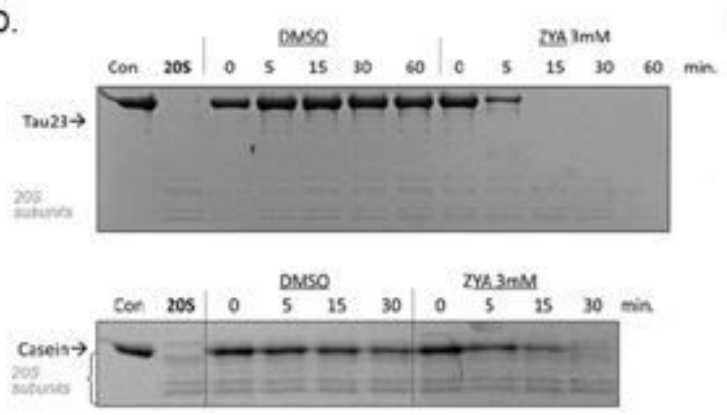

E.

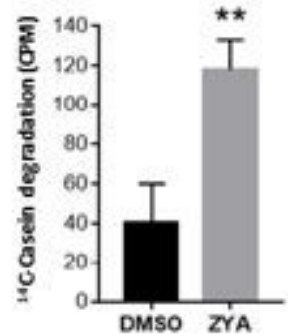

\section{Figure 5}

ZYA stimulates gate-opening in yeast and mammalian proteasomes. A. Dose response of ZYA with mammalian 20S proteasomes $(0.5 \mathrm{nM})$. Proteasome activity is normalized to DMSO control. Data is representative of three or more independent experiments performed in triplicate. Error bars represent \pm standard deviation. B. Wild type (WT) and gateless $(a 3 \Delta N)$ yeast 20 s proteasomes $(0.5 n M)$ incubated with or without ZYA $(2.5 \mathrm{mM})$ and $\mathrm{nLPnLD}$-amc. Experiment performed in triplicate. Error bars represent \pm standard deviation. C. Mammalian 20S proteasomes (0.5nM) incubated with ZYA (2.5mM), PA26 (1ug), neither or both. Proteasome activity measured using three substrates preferentially cleaved by different protease sites (LLVY-amc, chymotrypsin-like, nLPnLD-amc, capase-like; LRR-amc, trypsin-like). Experiment performed in triplicate. Error bars represent \pm standard deviation. D. Mammalian $20 S$ proteasomes $(100 \mathrm{nM})$ incubated with tau23 $(2 \mu \mathrm{M}$; truncated tau protein) or $\beta$-casein $(1 \mu \mathrm{M})$. At the indicated times, the reaction was quenched by addition of SDS loading buffer and separated by SDS-PAGE. Proteins visualized with Coomassie brilliant blue. Gels are representative of three independent experiments. 20S proteasome subunits are indicated with brackets to serve as loading controls for each sample. E. 14C casein was incubated with $20 \mathrm{~S}$ proteasome similar to D for $30 \mathrm{~min}$ at $37 \mathrm{oC}$. Acid soluble counts after TCA precipitation were quantified via scintillation. Error bars represent \pm standard deviation. 
A.

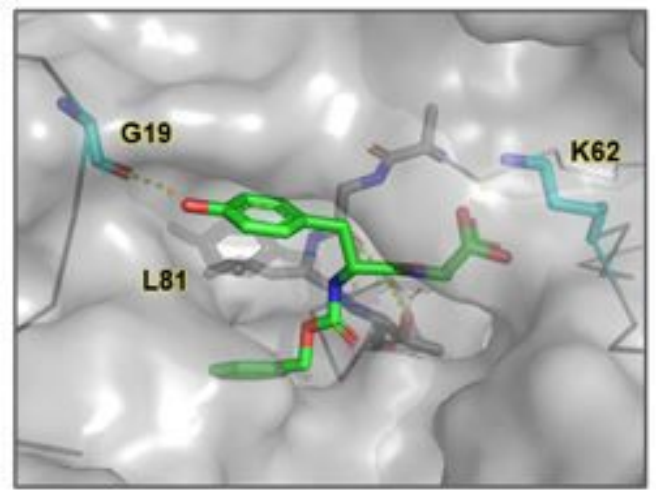

C.

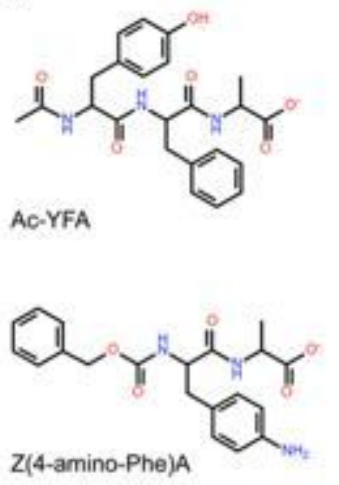

B.

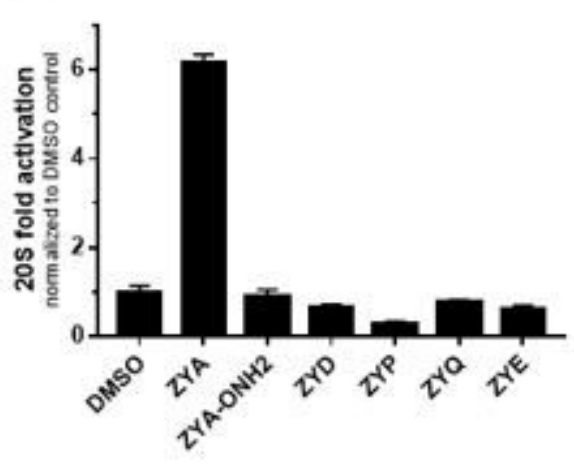

D.

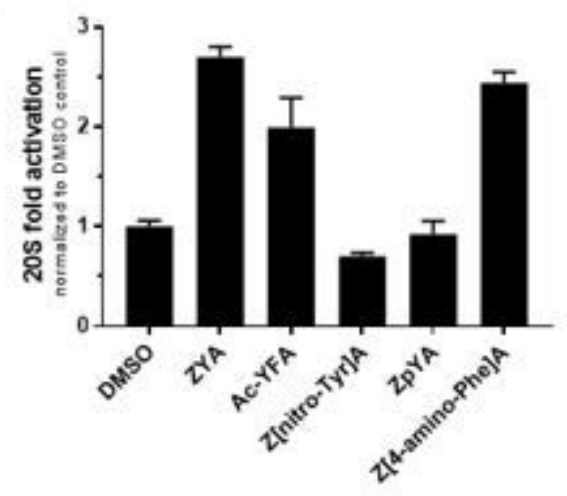

Figure 6

Effect of ZYA derivatives on proteasome gate activation. A. ZYA (green sticks) docked in intersubunit pocket between a5\&6 in human 20S. Image rendered in PyMOL. B. Mammalian 20S proteasome (0.5nM) activity (nLPnLD-amc hydrolysis, rfu/min) with the indicated ZYA derivatives at 500 $\mu$ M. Proteasome activity is normalized to DMSO. C. Structures of derivatives tested in D. D. Mammalian 20S proteasome activity (nLPnLD-amc hydrolysis, $\mathrm{rfu} / \mathrm{min}$ ) with the indicated ZYA derivatives at a low concentration of $100 \mu \mathrm{M}$. Proteasome activity is normalized to DMSO. Data are representative of three or more independent experiments performed in triplicate. Error bars represent \pm standard deviation. 

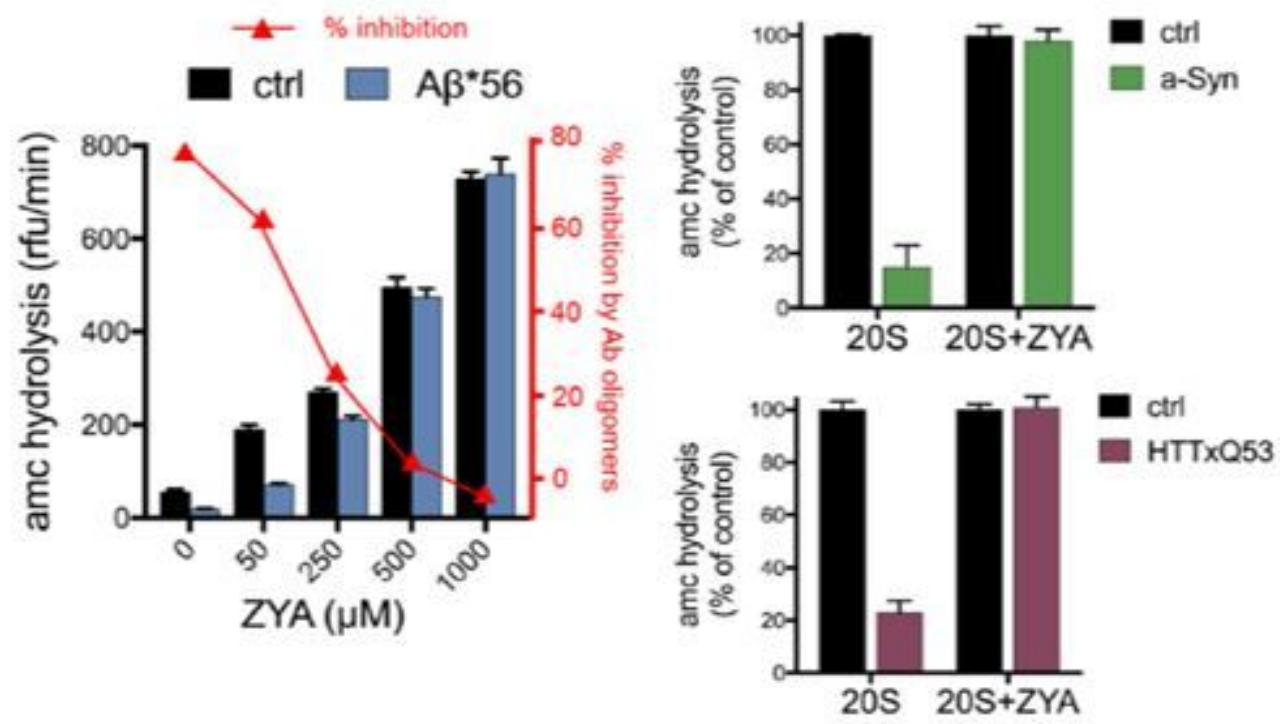

Figure 7

ZYA overcomes proteasomal impairment by ND-associated A11+ oligomers (Left) 20 S proteasome activity (nLPnLD-amc hydrolysis, rfu/min) with ZYA at the indicated concentrations, with and without $A b \star 56(0.5 \mathrm{mM})$ oligomers (Thibaudeau et al., 2018). (Right) 20S proteasomes (incubated with and without a-Syn (top) or HttQ53 (bottom) oligomers. Rate of nLPnLD-amc hydrolysis is normalized to the control. Data is representative of three or more independent experiments performed in triplicate. Error bars represent \pm standard deviation.

\section{Supplementary Files}

This is a list of supplementary files associated with this preprint. Click to download.

- SupplementaryFinal.pdf

- SupplementalMovie2.mp4

- SupplementalMovie4.mp4

- SupplementalMovie1.mp4

- SupplementalMovie3.mp4

- nrreportingsummaryChuah.pdf 